\title{
Analytical review of scientific theses defended in 2019 with the dissertation board D 999.063 .03 at the Russian Ilizarov Scientific Center for Restorative Traumatology and Orthopaedics of the Ministry of Health of Russia, FSBEI of higher education "South Ural State Medical University" of the Ministry of Health of Russia and FSBEI of higher education "Tyumen State Medical University" of the Ministry of Health of Russia
}

\author{
A.N. Diachkov', D.Yu. Borzunov ${ }^{2}$, Yu.P. Soldatov ${ }^{1}$ \\ ${ }^{1}$ Ilizarov National Medical Research Centre for Traumatology and Orthopedics, Kurgan, Russian Federation \\ ${ }^{2}$ Ural State Medical University, Ekaterinburg, Russian Federation
}

$\mathrm{PhD}$ and Doctoral candidates can submit theses/ dissertations to Dissertation Defense Committee D 999.063.03 in research specializations 14.01.15 traumatology and orthopaedics and 14.03.02 pathological anatomy.

Ten PhD and Doctoral theses were submitted and defended in 2019 including 2 Doctoral and 5 PhD dissertations in research specialization 14.01.15 traumatology and orthopaedics and $3 \mathrm{PhD}$ theses in research specialization 14.03.02 pathological anatomy.

Alexander V. Burtsev of FSGI «Russian Ilizarov Scientific Center «Restorative Traumatology and Orthopaedics» Ministry of Health of the Russian Federation submitted and defended Doctoral thesis on Surgical Stabilization Procedures for Surgical Pathology of the Cervical Spine in research specialization 14.01.15 traumatology and orthopaedics. Doctoral candidate adviser: Assistant Professor A.V.Gubin, Dr.Sci. (Med).

With 596 references reviewed, the applicant identified a broad spectrum of surgical disorders of the cervical spine and a variety of surgical stabilization techniques used to address the pathology. Manuals on diagnosis and treatment of spinal disorders describe over half of the pathologies affecting the cervical spine. This can be ascribed to the complexity and a variety of the disorders and treatment modalities used. The existing variety of nosologies and stabilization techniques interferes with the development of an appropriate systematization approach to evaluation and surgical treatment of cervical spine disorders and pose a challenge for practicing surgeons. There is a trend in today's health care environment with practice of referring patients with questionable diagnosis to allied health practitioners or other medical institutions (mostly federal centers) as well as anterior stabilization recognized as common and accessible versus posterior cervical fixation with pedicle screws being associated with potential risk factors. This is essential for paediatric patients with cervical spine anomalies and complicated nosological entities. The possibility with a posterior fixation using pedicle screws, in particular, is negated in some cases. Nevertheless, the international experience accumulated in surgical treatment of different cervical spine pathologies is evidence to the contrary.

The research aimed at optimization of diagnosis and treatment of surgical disorders of the cervical spine in patients of different age groups who required surgical stabilization. The applicant offered a syndromal approach to evaluation of cervical spine pathology to enable the development of clinical, diagnosis and treatment algorithms for preoperative planning of surgical stabilization in patients of different age groups identifying the role of posterior fixation in cervical spine surgery. A program has been developed for preoperative planning of posterior fixation involving advances in diagnostic imaging, computerized technologies and implants of the latest generation. Improvements offered and introduced into practice included a technique of freehand pedicle screw placement (utility patent № 2609776 of 03.02.2017); cervical screw placement using 3D-printing (patient-specific templates and 3D-models of cervical vertebrae). Grading scales used internationally to evaluate outcomes of spondylodesis following surgical stabilization of the cervical spine were critically assessed. Nosological entities were systematized to be applicable to surgical disorders 
of the cervical spine to allow the development of syndromal approach as a strategical concept.

A detailed description of posterior pedicle screw fixation techniques was provided in a step by step manner as a practical guide. Analysis of outcomes and workup findings allowed the author obtain new data on criteria of imaging evaluation to be considered in preoperative planning and further investigations.

The findings can be employed for trauma and orthopaedics decision-making processes in surgical stabilization of the cervical spine in patients of different age groups across all levels of institutions. Preoperative planning system was offered for posterior pedicle screw instrumentation. Techniques of pedicle screw placement were developed for specific localization of the pathology and the anatomy. Recommended criteria for assessing pedicle screw placement were developed with regard to localization and potential risks for vascular and nerve structures. Different assemblies of metal constructs were offered for surgical stabilization of transfer spine zones (craniocervical, axial-subaxial, cervicothoracic junctions). Recommended criteria for assessing long-term outcomes of surgical stabilization were developed for cervical spine surgery. Major findings of the research can be employed for decisionmaking of orthopaedic and trauma surgeons performing surgical stabilization of the cervical spine in patient of different age groups across all levels of institutions. There were 40 scientific publications brought out on the topic of the research.

Igor A. Komolkin of St.Petersburg State Pediatric Medical University, Ministry of Health of the Russian Federation submitted and defended Doctoral thesis on Pediatric Surgical Treatment of Congenital Chest Wall Deformities in research specialization 14.01.15 traumatology and orthopaedics. Doctoral candidate adviser: Professor A.Yu.Mushkin, Dr.Sci. (Med). The systematization of literature review of 370 sources on congenital chest wall deformities in children allowed identification of most important aspects involving:

- higher efficacy of current treatments of congenital chest wall deformities, objectivization of outcomes, causes of complications and adverse events;

- determining factors that would affect outcomes of congenital chest wall deformities exhibiting a variety of chest wall protrusion and very diverse clinical manifestations, age at appearance and specific features of progression;

- genetic factors for prognosis of the course of most common forms of congenital chest wall deformities;

- effect of deformed rib cage on the shape and dimensions of heart chambers and possible disorders of intracardiac hemodynamics;

- emotional and individual attributes of children with most common types of congenital chest wall deformities.
With a diversity of surgical techniques available for treatment of congenital anomalies of chest wall there is a high risk of complete or partial recurrence that requires the development of new methods for reconstruction of the thoracic spine, the ribs and the sternum. The applicant undertook to systematize anatomical variants of congenital chest wall maldevelopment considering the spatial orientation and develop an objective quantitative criteria to assess severity of the condition and efficacy of the treatment. The purpose of the research was to develop a system of pediatric surgical treatment of congenital chest wall deformities using threeplanar classification of congenital anomalies of the ribs and the sternum, techniques of quantitative assessment, differential indications to surgical treatment and methods of operative correcion. The applicant systematized data on different types of congenital chest wall deformities in children including rare and syndromal conditions developing strategical scheme (classification). Genes and regulatory elements of bone tissue having a role in the development of most common types of congenital chest wall deformities were identified in the patients. An $\mathrm{h}$ allelic gene, hh genotype, in particular, was shown to be a marker of severe and progressive deformities in patients with funnel chest deformity. Emotional and individual attributes of children with funnel chest and keel chest were identified including the postoperative appearance. Psychological follow-up was found to be an essential component for successful social adaptation of the patients at stages of the treatment. Benefits of surgical reconstruction of the thoracosternal and thoraco-sterno-spinal entities were shown during treatment of congenital chest wall deformities including lower risk of errors and complications and their correlation between the type of anomaly, patient's age and severity of the condition. Integrated quantification index was devised for objectivization of outcomes of surgical treatment of rare chest wall deformities and their severity. The applicant and coauthors offered new technical devices and techniques for surgical treatment of children with congenital chest wall deformities: «Technique of surgical treatment of funnel chest deformity» (Patent RF № $2472452 \mathrm{dtd}$ 03.10.2011); «Plate for stabilization of thoraco-sternal entity» (Patent RF №112617 dtd 26.07.2011); «Device for modeling a plate for correction of the funnel chest deformity» (Utility patent RF №111745 dtd 12.05.2011); «Technique of preoperative planning for funnel chest deformity and compression of the heart» (Patent RF № 2489182 dtd 16.06.2011). Differential indications to surgical treatment and methods of pediatric operative correcion of congenital chest wall deformities were identified taking into consideration patient's age and severity of 
the condition to prevent tactical and technical failures and choose an optimal surgical option. New indices were offered and the known quantification indices (hemithorax asymmetry, chest compression, vertical chest asymmetry) were upgraded for objectivization of severity of the pathology and outcomes of surgical treatment of patients with deformities of thoracosterno-spinal entities. There were 30 scientific publications brought out on the topic of the research.

Olga M. Sergeenko of FSGI «Russian Ilizarov Scientific Center «Restorative Traumatology and Orthopaedics» Ministry of Health of the Russian Federation submitted and defended $\mathrm{PhD}$ thesis on Diagnosis and Surgical Treatment of Atlantoaxial Dislocation Secondaryto Congenital Craniovertebral Junction Anomaly in research specialization 14.01.15 traumatology and orthopaedics. Doctoral candidate adviser: Assistant Professor A.V. Gubin, Dr. Sci. (Med).

With 283 references of Russian and foreign authors reviewed, the applicant explored diagnosis and treatment of atlantoaxial dislocation (AAD) secondary to congenital craniovertebral junction (CVI) anomalies. Congenital AAD is reported to be associated with such orthopaedic problems as destabilized cervical spine and rigid deformities. Singular cases or small case series are reported in the literature. The congenital AAD associated mortality rate is unknown with the incidence rate of myelopathy and medullopathy ranging between $40 \%$ and $100 \%$. Most of the patients with cervical spine anomalies are treated in the leading research trauma and orthopaedic centers of the Russian Federation using a comprehensive orthopaedic approach and assistance of related specialist, specialized organizational arrangement and staff training programs. Although a satisfactory functional and cosmetic result can be obtained with timely diagnosis and current treatment of congenital AAD with a lower risk of neurological complications, literature review indicates to a paucity of studies on the issue and the absence of reliable diagnosis and treatment algorithms. The purpose of the research was to improve outcomes of pediatric surgical treatment of AAD secondary to congenital CVI anomalies using new clinical and diagnosis algorithms and surgical approaches. Differential indications to a variety of surgical techniques used to treat patients with AAD secondary to congenital CVJ anomalies were identified taking into consideration physical and radiological findings of the anomaly and surgical strategy worked out for efficacious management. Preoperative and intraoperative patient preparation technique was devised with the use of halo-traction to improve patient's mobility and primary reduction of $\mathrm{AAD}$ providing adaptation of spinal circulation and prevention of neurologic complications. An original algorithm was devised for diagnosis and the choice of treatment strategy for pathological AAD of congenital etiology and unknown genesis. A system of comprehensive examination was developed, clinical and radiological criteria identified for optimal surgical strategy. Classification systems of pathological AAD of congenital etiology and unknown genesis were optimized for practical use. Indications to halo-traction in patients with pathological AAD of congenital etiology were specified and extended to ease the surgical stage of treatment and decrease a risk of postoperative neurological complications. Optimal quantitative and qualitative grading scales were identified for assessment of neurological and functional status of pediatric patients with pathological $\mathrm{AAD}$ of congenital etiology and unknown genesis treated surgically or conservatively. There were 6 scientific publications brought out on the topic of the research in Russian journals indexed within the database of State Commission for Academic Degrees and Titles and 2 articles in peer reviewed journals («Journal of craniovertebral junction and spine», «World Neurosurgery»).

Ramil R. Bidyamshin of FSGI «Russian Ilizarov Scientific Center «Restorative Traumatology and Orthopaedics» Ministry of Health of the Russian Federation submitted and defended $\mathrm{PhD}$ thesis on Palliative Orthopaedic Surgical Interventions for Hip Dislocation in Children with Cerebral Palsy in research specialization 14.01.15 traumatology and orthopaedics. Doctoral candidate adviser: D.A.Popkov, Dr.Sci. (Med), Professor of RAS.

Literature review of 118 sources showed that hip displacement is very common in children with cerebral palsy (CP) classified as GMFCS levels IV and $\mathrm{V}$ ranging between $2.6 \%$ and $75 \%$ and associated with poor quality of life, the development of coxarthrosis and pain, malaligned femur, difficulties in comfortable posturing and hygiene. The pathology results in considerable impairment of functional capacity, trophic disorders of investing tissues, lost capacity of passive verticalization, poor socialization, severe osteoporosis and decompensation of inner organ conditions. The available surgical treatment is mostly palliative and aimed at elimination or relief of pain, improvement of comfortable sitting posture and hygiene. Surgical options include extraperiosteal resection of the proximal femur and interposition of soft tissues between the femoral shaft and the pelvis, valgus osteotomy with/without femoral head resection. Total hip arthroplasty being a reliable option for coxathrosis of different etiology was shown to be effective rfor $\mathrm{CP}$ children classified as GMFCS levels I, II and III.

Literature review of the last 25 years allowed the applicant to conclude that outcomes of palliative procedures were evaluated using the above three 
criteria. According to some Russian and foreign researchers no substantial differences in the efficacy of the procedures have been detected. Outcomes of the treatment have not been assessed with regard to severity of motor disorders and extent of surgical trauma. Another important lacking criteria for the existing approach is passive verticalization of the patients as one of the most essential aspects of quality of life. There have been no publications on palliative procedures reported as a single event surgery performed with surgical correction of the knee and ankle contractures and foot deformities as well as evaluation of the function and quality of life at a long term. The purpose of the research was to improve outcomes for adolescent and adult patients with non-reconstructable hip displacement suffering from severe type of CP based on studies on efficacy of palliative orthopaedic procedures and substantiation of differential surgical approachs to multi-level single-event surgery. The review of short- and longterm follow-ups of 41 patients showed that indications to palliative interventions produced for $\mathrm{CP}$ patients with non-reconstructable hip displacement included creating conditions for passive verticalization of the patients with weight-bearing on the lower limbs in addition to well established indications (pain control, elimination of femur malalignment, improvement of conditions for hygiene, sitting tolerance). Palliative interventions (arthroplastic resection of the proximal femur and proximal support osteotomy) used by the author were shown to be effective for pain relief and elimination of malaligned femur. The applicant suggested that the pelvic support osteotomy can provide conditions for reliable passive verticalization. Simultaneous elimination of the flexion tibial alignment, contracture of the ankle joint, foot deformities is an obligatory prerequisite for restoring weight-bearing capacity of the lower limbs and improving quality of life. Addressing the orthopaedic problems with concurrent palliative hip surgery facilitates improved functional (passive and active) capabilities for the patients and quality of life. The own modification of support osteotomy of the proximal femur (patent RU 2620358 dtd 29.03.2016) can provide greater arc of motion for abduction and flexion of the femur compared to classical McHale method maintaining the possibility with axial support on the limb and verticalization of patients.

Surgical technique of palliative surgeries, modified procedures, expected outcomes, probable errors and complications, ways of the their prevention and treatment are described in details in the thesis. Multi-level single-event surgical procedures on lower limbs are substantiated and demonstrated in the study with the core palliative hip surgery aimed at increase in passive and active functional capabilities of CP patients with severe neurological disorders and limited motor prognosis. There were 13 scientific publications brought out on the topic of the research including 5 in journals indexed within the database of State Commission for Academic Degrees and Titles and 2 articles in Scopus indexed journals, 1 manual and 1 patent received.

Edward R. Mingazov of FSGI «Russian Ilizarov Scientific Center «Restorative Traumatology and Orthopaedics» Ministry of Health of the Russian Federation submitted and defended $\mathrm{PhD}$ thesis on «Elastic intramedullary nailing and combined osteosynthesis in lower limb deformity correction in patients with severe osteogensis imperfecta (clinical and experimental study» in research specialization 14.01.15 traumatology and orthopaedics. Doctoral candidate adviser: D.A. Popkov, Dr. Sci. (Med), Professor of RAS. With 101 references reviewed, the applicant explored osteogenesis imperfecta (OI) as a group of genetic disorders with different types of inheritance characterized by fragile bones that break easily, leading to associated deformities, low bone mineral density and osteopenia. In most cases (at least $70 \%$ ) OI is caused by mutations in the COL1A1 gene or COL1A2 gene. In 2015 the Russian Registry of IO cases included 650 patients (among them 421 children). Considering prevalence of the condition worldwide, the expected number of cases can amount to 12.5-25 thousands. Major orthopaedic manifestations of $\mathrm{OI}$ are decreased mineralization and mechanical skeletal strength, fractures and deformities of long bones, skull deformity and hypermobility of the joints. Recurrent fractures, limb deformities, continuous periods of immobilization and absence of weight-bearing contribute to low BMD, motor skills maldevelopment, acquisition of verticalization skills and self-care. Somatic and functional development is delayed in children with severe and moderate OI. Treatment of patients with OI is aimed at prophylaxis of fractures and deformities using bracing, bisphosphonate therapy, preventive intramedullary telescopic nailing and correction of long bone deformities. Intramedullary nailing is primarily used for fixation of broken bones or deformity correction if nonoperative treatment is not indicated. As a self-extending system telescopic rodding of long bones in children with OI allows reinforcement of the whole bone all along the length whilst growth occurs. Rigid or elastic rods are transepiphyseally introduced in the intramedullary canal. However, telescopic intramedullary rodding has some disadvantages. There are controversial aspects in application of elastic and telescopic rods including technical difficulties and high costs of the latter. Resurgery rate can be as high as $50 \%$ with application of telescopic rods and $58-87 \%$ with elastic 
rods in achievement of the appropriate result. An effect of transphyseal nailing on longitudinal growth of the bone has not been identified in patients with OI. No algorithm of differential application of techniques has been developed with regard to patient's age and type of OI. Optimal terms of external fixation have not been determined with use of combined techniques, and complications have not been systematized. So those were included in the objectives of the research.

The purpose of the research was to improve results of surgical treatment of patients with severe OI based on the study of the efficacy and substantiation of the differential application of elastic intramedullary nailing and combined osteosynthesis in correction of lower limb deformity. The novelty of the research includes an effect of transphyseal nailing on bone growth and formation of articular ends identified in patients with OI. Transphyseal elastic nailing has been shown to result in 15-18 \% loss of longitudinal residual growth of the segment. Eccentrical placement of transphyseal rods leads either to the development of angular deformity at the growth or delayed physiological orientation of the articular surface. Intramedullary and subperiosteal components being integrated into the bone, the newly formed compact bone, in particular, are meant to function for reinforcement, and subperiosteal reinforcement does not delay bone consolidation. Practical relevance of the research includes the suggestion, substantiation and identification of indications to the use of new techniques of surgical correction of lower limb deformities in children with severe OI and modifications of the known method of transphyseal intramedullary reinforcement with elastic nails. Differential application of techniques for correction of lower limb deformities depending on patient's age and severity of OI using intramedullary (transphyseal for children) nailing as a major surgical component was shown to provide a prolonged increase in mechanical bone strength improving patient's ambulation and quality of life. There were 11 scientific publications brought out on the topic of the research including 3 in journals indexed within the database of State Commission for Academic Degrees and Titles, 3 in Scopus indexed journals and journals indexed within the database of State Commission for Academic Degrees and Titles, and 3 articles in Scopus indexed journals, one chapter in a book, one article, one manual and a patent received.

Egor Yu. Filatov of FSGI «Russian Ilizarov Scientific Center «Restorative Traumatology and Orthopaedics» Ministry of Health of the Russian Federation submitted and defended $\mathrm{PhD}$ thesis on «Surgical treatment of patients with congenital spine deformities secondary to isolated and multiple vertebral anomalies with leading monosegmental component» in research specialization 14.01.15 traumatology and orthopaedics. Doctoral candidate adviser: S.O. Ryabykh, Dr. Sci. (Med). With 206 references reviewed, the applicant explored spine deformities secondary to congenital anomalies including a large group of patients with a variety of vertebral malformations. Treatment options for congenital spine anomalies are well described in the Russian and foreign literature to include a variety of instrumentation fixation for definitive local boneplasty spondylodesis or dynamic correction of spine deformity. More effective and more aggressive techniques for correction of local congenital deformity are employed with the use of different vertebrotomies to extirpate the abnormal vertebra applying anterior and posterior instrumentation fixation. There is no uniform methodological approach to the treatment of patients with congenital spine deformity described in the literature. There are no accurate data on substantiation of a definitive technique of osteotomy and identification of instrumentation site as well as Cochrane recommendations and systematic reviews that would clarify the issue. The factors gave reason for the reasearch.

The purpose of the research was to improve results of surgical treatment of patients with congenital spine deformities with leading monosegmental component using differential surgical strategy. The applicant performed a systematic review of surgical interventions produced for congenital spine deformities with leading monosegmental component, identified errors and complications with different approaches to the treatment of the above condition and their prophylaxis. Advantages of dorsal approach to correction of congenital spine deformity with leading monosegmental component were demonstrated as a universal approach to the treatment of the pathology. Three column pedicular asymmetrical osteotomy outside the apical zone was offered for correction of spine deformity secondary to combined and monosegmental vertebral anomalies of a 'complicated anatomical localization'. The benefits of pedicular approach were shown in correction of congenital spine deformities with leading monosegmental component. Individual approaches to surgical treatment of patients with spine pathology secondary to monosegmental vertebral anomalies were developed considering patient's age and such essential features of vertebral syndrome as the type of monosegmental anomaly, the localization and the size of the deformity. A new variant of surgical correction of spine deformity secondary to monosegmental vertebral anomalies of a 'complicated anatomical localization' was developed and its clinical relevance demonstrated. There were 14 scientific publications brought out on the topic of the research including 4 articles in journals indexed within the database of State Commission for Academic Degrees and Titles. 
Alexandra A. Rogozina of FSGI VO «South Urals State Medical University» Ministry of Health of the Russian Federation submitted and defended $\mathrm{PhD}$ thesis on "Age related clinical and morphological manifestations of endometrial polyps in women» in research specialization 14.03.02 pathological anatomy. Doctoral candidate adviser: Assistant Professor E.E.Voropaeva, Dr.Sci. (Med). With 133 references reviewed, the applicant explored the problem of endometrial polyps (EP) being relevant for current pathological anatomy and gynecology. Polyps of the endometrium are among the common pathologies of the reproductive organs and can be a cause of infertility in women, abnormal genital bleeding, chronic pubic pain that necessitate intrauterine manipulations causing an injury to endometrium and uterine synechias. The applicant reviewed classifications and histological diagnosis of EP described in fundamental investigations. However, prevalence and patterns of EP have not been reported in patients of different ages. No comprehensive clinical and pathomorphological characteristics of different morphological types of EP evaluating endometrium in this pathology have been described as well as age related immunohistochemical manifestations of different morphological types of EP. Greater prevalence of recurrent EP necessitates the development of personified approaches to the treatment strategy for patients with EP, different morphological types taking into consideration clinical, anamnestic and histological findings of EP and the endometrium as well as immunohistochemical characterization of EP.

The purpose of the research was to identify clinical and morphological manifestations of endometrial polyps in women of different age. The applicant identified prevalence and patterns of EP in patients of different ages. The rate of EP was found to be greater in postmenopausal women (44.2 \%) than in women at late reproductive age (23.3\%), menopausal transition age $(19.2 \%)$ and early reproductive age $(13.3 \%)$. Morphological patterns included atrophic, functional and hyperplastic EP. The rate of atrophic and mixed EP was significantly higher in postmenopausal women; functional EP prevailed in women at late reproductive age. Hyperplastic EP had approximately similar distribution in women at late reproductive age, postmenopausal women, menopausal transition age with lower rate at early reproductive age. Adenomyomatosis EP were more common in older women. EP of different morphological types had clinical pathomorphological characteristics. Infertility and symptom free course of disease are common for functional EP. Abnormal genital bleeding and hypogastrium pain were common for atrophic, hyperplastic and mixed EP. Functional EP were represented with proliferative forms in $66.1 \%$ in women at reproductive age and $100 \%$ in women at menopausal transition age. Low profile chronic endometritis or inactive condition with fibrosis were observed in $73.1 \%$ of atrophic EP and endometrium. Every second patient developed chronic inflammation in the polyp in hyperplastic EP and low profile moderate inflammation in the endometrium in $64.5 \%$ of the cases. Adenomyomatosis EP were characterized by smooth myocytes with indifferent endometrium (66.7 \%) with no signs of inflammation. Mixed EP contained endometrial and endocervical glands in the fibrous stroma. Signs of reactive inactive inflammation were observed in the endometrium in $80.0 \%$ of the cases.

Immunohistochemical characteristics of EP were identified. Expression of ER and Ki67 was significantly higher than in the functional EP specimen of secretory types and was significantly higher in hyperplastic EP than in proliferative types of functional EP. Expression of p16INK4a oncoproteins in EP was observed in every fifth case of functional EP and every fourth case of hyperplastic EP with three-fold increase in hyperplastic EP at menopausal transition age as compared to functional EP $(p=0.01)$ and five-fold increase at menopause $(p=0.001)$. There was no expression of p16INK4a in atrophic EP, and expression of ER, PR, Ki67, Bcl2 were significantly lower than those in functional EP of proliferative type. No specific features in expression of ER, PR, Ki67, $\mathrm{Bcl} 2$, p16INK4a were revealed in adenomyomatosis and mixed EP. There were 6 scientific publications brought out on the topic of the research including 3 articles in Russian peer reviewed journals, one manual, one article in peer reviewed research journal, one article in abtract book of a regional meeting.

Svetlana A. Gordeeva of FSGI VO «South Urals State Medical University» Ministry of Health of the Russian Federation submitted and defended $\mathrm{PhD}$ thesis on «Clinical and morphological manifestations of refraction anomaly in children" in research specializations 14.03 .02 pathological anatomy, 14.01 .07 eye diseases. Doctoral candidate advisers: Professor E.L. Kurenkov, Dr. Sci. (Med), Assistant Professor V.S. Rykun, Dr. Sci. (Med). With 238 references reviewed, the applicant found that around 285 million people worldwide suffer from eye disorders including 19 million children. From them, 12 million children suffer from refraction anomalies associated with typical morphological reactions related to remodeling of the fibrous tunic of the eyeball and Tenon's capsule. Changes in cells, extracellular matrix, fibroblast ultrastructure are observed in the Tenon's capsule in ametropy cases. Clinical manifestations of ametropies include changes in the optic biometry and in the tissues of the eyeball. 
Myopy is a prevalent disease among childhood pathologies of the eye and adnexa constituting $34 \%$. According to E.S. Libman (2006) steady increase in childhood disabilities caused by eye disorders due to refractive errors has medical and social role, and children experience difficulties in developing social skills. Although pathogenesis of myopia is associated with increased intraocular pressure, changes in biomechanical properties of sclera, specific features of extracellular matrix, cellular composition, fibroblast ultrastructure of the Tenon's capsule have not been explored in children suffering from different types of clinical refraction. Tunica fibrosa oculi is involved in refraction errors, and biometrical characteristics, electronic microscopic manifestations of the fibrous capsule of the eye are essential for understanding the morphological manifestations of progressing myopia. Scleral structure appears to alter in progressing myopia with increased anteroposterior axis of the eye ball. According to some authors, pathological processes developing in the sclera are similar to those occurring in the Tenon's capsule. The processes developing in the Tenon's capsule can be extrapolated to the sclera due to common histogenesis and uniform morphological, chemical and functional characteristics. A comprehensive clinical and morphological characterization of refraction errors in children with changes in the fibrous capsule of the eye with progressing myopia has not been fully represented in the literature. Cellular composition and fibroblast ultrastructure have been understudied in morphological aspect as well as the structure of extracellular matrix of the Tenon's capsule in different types of clinical refraction in children.

The purpose of the research was to perform comprehensive clinical and morphological research of refraction anomalies in children to improve morphological diagnosis of ametropy and prognosis for progression of myopia. The applicant explored fibroblast unltrastructure of the Tenon's capsule in different types of clinical refraction in children. Hyperplasia and hypertrophy of the synthetic and power-producingapparatuses of thecellswereidentified in progressing myopia, and in contrast, atrophic fibroblast organoids were noted in hypermetropy. Progressing myopia and hypermetropy have been studied in adaptation aspects with the structural base of hyperplasia and atrophy, respectively. Morphometric analysis was produced in pediatric refraction errors to measure cells in the Tenon's capsule. Progressing myopia was characterized by increased fibroblasts and adipose cells with cellular hyperplasia detected as an adaptation sign. Ultrastructural fibroblast morphometry of the Tenon's capsule revealed intracellular hyperplasia of organoids in progressing myopia and their atrophy in hypermetropy. Cellular reaction of the Tenon's capsule on the growth of the eye ball was explored and clinical characteristics of the eye ball compared: anteroposterior axis (APA), gradient of the annual progression with changes in the structure of the Tenon's capsule of the eye ball. With the APA of the eyeball increased by more than $24.50 \mathrm{~mm}$ adipocytes were shown to decrease; with the APA increased by more than $24.90 \mathrm{~mm}$ adipocytes were shown to increase; with the APA increased by more than $26.50 \mathrm{~mm}$ the maximal length of the fibroblast processes and low density of collagen fibrills were observed. A technique for prognosis of the progression of myopia was offered for the first physical examination of the patient with the equation predicting progression of myopia with the probability of $81.8 \%$. Equation of regression was established for diagnosis of ametropy type based on morphometric analysis of the Tenon's capsule. Number of fibroblasts in the Tenon's capsule apperaed to be a morphological marker for detection of refraction error. The criteria allowed for verification of optical diagnosis with accuracy of $85.1 \%$. A technique for prognosis of the progression of the operated myopia was offered with the equation enabling prognosis of the progression of the operated myopia with the probability of $87.5 \%$. General pathological reaction in the Tenon's capsule in refraction errors was accompanied by hyperplastic processes in progressing myopia and atrophic changes in hypermetropy. There were 12 scientific publications brought out on the topic of the research including 7 articles in journals indexed within the database of State Commission for Academic Degrees and Titles; patent № 2621944 dtd 08.06.2017 «Technique for prognosis of the progression of myopia in children» obtained.

Roman S. Maslov of FSGI VO «South Urals State Medical University» Ministry of Health of the Russian Federation submitted and defended $\mathrm{PhD}$ thesis on «Pulmonary embolism: clinical and pathoanatomical characteristics and comparisons using autopsy samples» in research specializations 14.03.02 pathological anatomy. Doctoral candidate advisers: Assistant Professor A.A.Dub, Dr.Sci. (Med). With 55 references reviewed, the applicant found that pulmonary embolism (PE) is the third most common cause of cardiovascular death worldwide after stroke and heart attack and is responsible for an estimated $0.1 \%$ deaths worldwide. The European Society of Cardiology identified risk factors as primary (familial) and secondary (acquired). The nature and combination of secondary risk factors as the cause of PE including diseases, symptoms, sympomocomplexes and iatrogenic pathology have a substantial scientific and practical role and require additional investigation. Most patients who succumb to PE do so within the first few hours and the first day of the event without 
adequate treatment with mortality rate of more than $30 \%$. Evaluation of prophylaxis and treatment of PE is to be considered with the quality of medical assistance provided. Literature review indicated to a need of a research and specification of morphological aspects and development of a conditioned approach to the analysis of nosological entities associated with PE.

The purpose of the study was to identify causes of venous thromboembolism in adults of different age groups and inpatient hospital death rates in PE establishing clinical and morphological manifestations of the complication based on retrospective and prospective clinical and pathoanatomical evaluation of autopsy samples. The applicant showed that adequate judgement of the rate of $\mathrm{PE}$, a life-threatening complication, can rely on the findings of thanatological (clinical and pathoanatomical) examination. Propositions of current conditionalism and ICD-10 can be considered as the base for establishing the cause of mortality in PE. Bi- and multicausal genesis of death is common for retired patients as compared to those of working age. The nature of rheological pulmonary disorders is identical for different causes. PE normally occurs with evident signs of polyorganic, pulmonary and cardic insufficiency. PE is clinically recongnized in governmental and municipal hospitals in $48 \%$ only with recommended prophylaxis and treatment administered in $7 \%$ of the reviewed observations. The inpatient hospital PE related death rate at governmental and municipal hospitals of the Chelyabinskaya Oblast was reported to be as high as $8.98 \%$ per year. The inpatient hospital PE related death rate was higher in retired men with the reported incidence of $12.8 \%$ while the average death rate was $6.4 \%$ in patients of working age. Based on clinical and morphological manifestations PE was shown to result from a combination of different nosological entities as well as iatrogenic diseases and other critical and terminal conditions termed as secondary risk factors for PE. This served the base to use propositions of current conditionalism and ICD-10 for establishing the cause of mortality in PE. Clinical and morphological characterization of PE and mortality associated with other critical and terminal conditions included a wide range of rheological pulmonary changes but progression of polyorganic insufficiency in the form of pulmonary and cardiac failure was found to cause PE by thrombosis of smaller rami of the main pulmonary artery in $30-50 \%$ of the cases in presence of thromboemboli in major and smaller rami of different duration. Fatal outcomes were common in evident sclerotic and alternative changes in the organ in combinations with different nosological entities in deaths caused by PE or other pulmonary complications, and sclerotic pulmonary vessels and perivascular respiratory tissues were more common for PE as compared to other complications. More information was provided on causes of PE, defects of health care, shortcomings of the prophylaxis as well as the higher rate of PE in retired individuals. $\mathrm{Bi}$ - and multicausal genesis of diseases and death was shown to be important for thanatogenesis of PE in the analysis of secondary risk factors of the complication. This led to substantial difficulties in diagnosis and treatment of the complex combination of terminal and critical conditions of the challenging pathology. There were 13 scientific publications brought out on the topic of the research including 4 articles in Russian peer reviewed journals, one manual for students and postgraduates, physicians of different specialties.

\section{Information about the authors:}

1.Alexander N. Diachkov, M.D., Ph.D., Professor, Ilizarov National Medical Research Centre for Traumatology and Orthopedics, Kurgan, Russian Federation

2.Dmitry Yu. Borzunov, M.D., Ph.D.,

Ural State Medical University, Ekaterinburg, Russian Federation,

Email: borzunov@bk.ru

3. Yuri P. Soldatov, M.D., Ph.D., Professor, Ilizarov National Medical Research Centre for Traumatology and Orthopedics, Kurgan, Russian Federation 